\title{
Ultralong single-wall carbon nanotubes
}

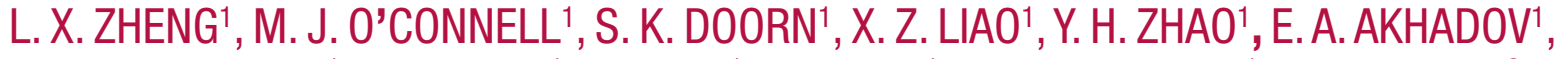 \\ M. A. HOFFBAUER ${ }^{1}$, B. J. ROOP' ${ }^{1}$, Q. X. JIA ${ }^{1}$, R. C. DYE¹, D. E. PETERSON¹, S. M. HUANG² , J. LIU² \\ AND Y. T. ZHU ${ }^{1 *}$ \\ ${ }^{1}$ Los Alamos National Laboratory, Los Alamos, New Mexico 87545, USA \\ ${ }^{2}$ Chemistry Department, Duke University, Durham, North Carolina 27708, USA \\ *e-mail:yzhu@lanl.gov
}

S ince the discovery of carbon nanotubes in 1991 by lijima $^{1}$, there has been great interest in creating long, continuous nanotubes for applications where their properties coupled with extended lengths will enable new technology developments ${ }^{2}$. For example, ultralong nanotubes can be spun into fibres that are more than an order of magnitude stronger than any current structural material, allowing revolutionary advances in lightweight, high-strength applications ${ }^{3}$. Long metallic nanotubes will enable new types of microelectromechanical systems such as micro-electric motors, and can also act as a nanoconducting cable for wiring micro-electronic devices ${ }^{4}$. Here we report the synthesis of 4-cm-long individual single-wall carbon nanotubes (SWNTs) at a high growth rate of $11 \mu \mathrm{m} \mathrm{s}^{-1}$ by catalytic chemical vapour deposition. Our results suggest the possibility of growing SWNTs continuously without any apparent length limitation.

Figure la shows the photograph of a 4.8 -cm-long Si substrate and a curve formed by superimposing on it 230 scanning electron microscopy (SEM) images taken along an individual 4-cm-long SWNT. As shown later, only SWNTs were observed in our samples. Figure $1 \mathrm{~b}-\mathrm{d}$ shows the 4-cm SWNT at its beginning, middle and end, respectively. Note that the 4-cm-long SWNT tangled up near the substrate edge (Fig. 1d), possibly with other SWNTs, making it impossible to follow the SWNT to its actual end. Therefore, we assume that it terminated where it can no longer be followed (marked by an arrow in Fig. 1d). We observed several long SWNTs terminating at either the substrate end or side edges, suggesting that the SWNT length is only limited by the substrate size. In other words, there might be no limitation to the SWNT length if the Si substrate were sufficiently long and wide. However, our substrate size was limited by the sample chamber size of the scanning electron microscope (SEM; JEOL 6300FXV) that we are using to characterize the SWNTs. The full image of the $4-\mathrm{cm}$ SWNT is shown in the Supplementary Information.

The ultralong SWNTs were synthesised by Fe-catalysed decomposition of ethanol. A $0.1 \mathrm{M} \mathrm{FeCl}_{3}$ solution was applied with a dip-pen to one end of the Si substrate, which was then placed in a horizontal $2.5 \mathrm{~cm}$ quartz tube furnace with the catalyst end directed toward the gas flow. The catalyst was calcined at $900{ }^{\circ} \mathrm{C}$ for 30 minutes under a flowing $\left(30 \mathrm{~cm}^{3} \mathrm{~min}^{-1}\right) \mathrm{Ar}+6 \% \mathrm{H}_{2}$ gas mixture. Ethanol vapour was then introduced into the furnace by bubbling the same gas mixture through ethanol at a flow rate of $20 \mathrm{~cm}^{3} \mathrm{~min}^{-1}$. The growth was typically
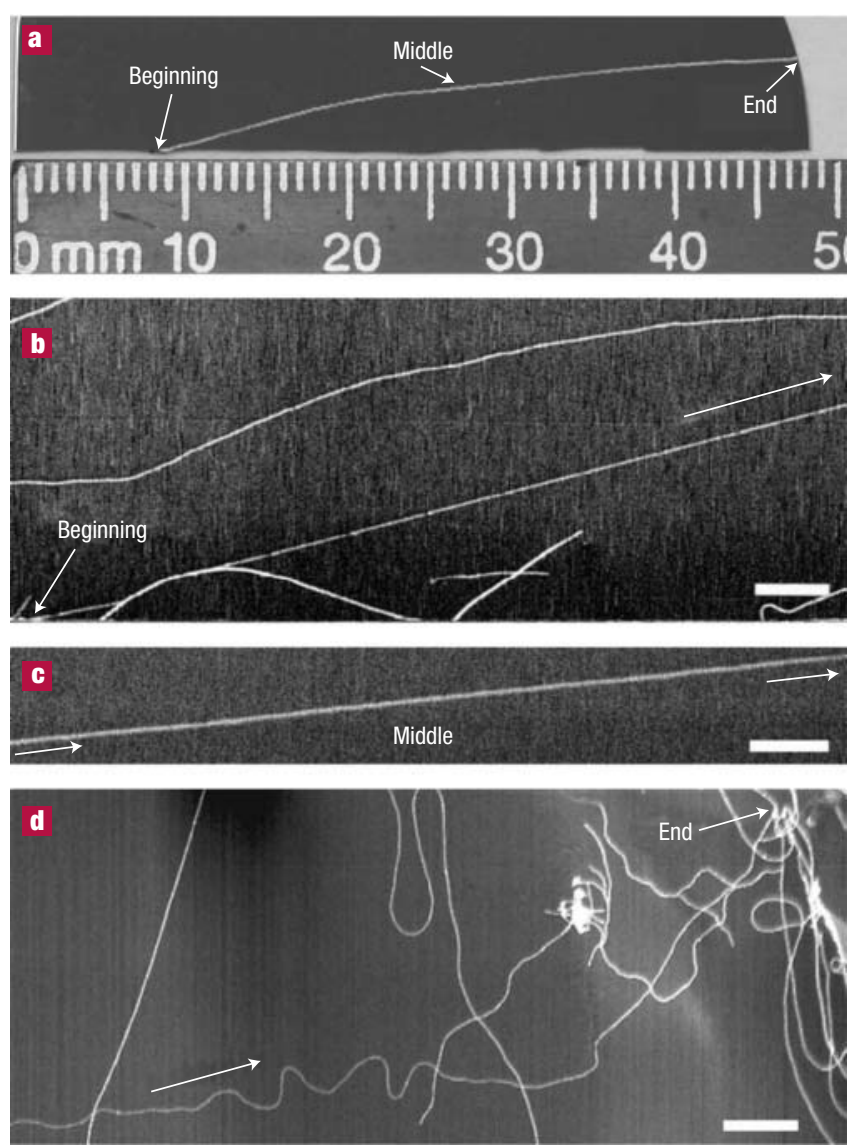

Figure 1 A 4-cm-long carbon nanotube. a, Photograph of a 4.8-cm-long Si substrate on which a 4-cm SWNT was synthesised. The curve was formed by superimposing on the photograph 230 scanning electron microscopy (SEM) images along the individual 4-cmlong SWNTs. $\mathbf{b}$, SEM image of the beginning segment of the SWNT. c, SEM image of a middle segment as marked by an arrow in Fig. 1a.d, SEM image of the end segment. See the Supplementary Information for the complete image of the 4-cm-long SWNT. The scale bars are $10 \mu \mathrm{m}$. 

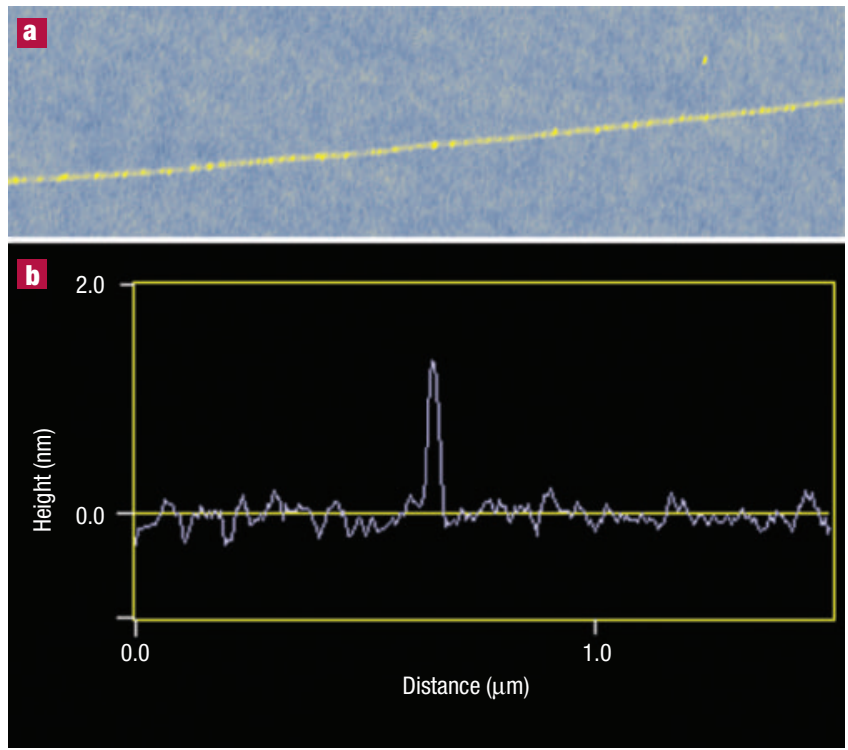

Figure 2 AFM. a,AFM image of a segment of the 4-cm-long SWNT (image width: $5 \mu \mathrm{m}$ ) b, The height profile across the 4-cm SWNT, with its diameter determined from the peak heightas $1.4 \mathrm{~nm}$.

terminated after $1 \mathrm{~h}$ by switching the gas to the $\mathrm{Ar}+6 \% \mathrm{H}_{2}$ gas mixture and cooling down the furnace.

The SWNTs grew at extremely high rates. Assuming that the $4-\mathrm{cm}-$ long SWNT grew over the entire 1-h synthesis duration, the growth rate can be calculated as $11 \mu \mathrm{m} \mathrm{s}^{-1}$, which is several times higher than a previously reported ${ }^{5}$ high growth rate $\left(3 \mu \mathrm{m} \mathrm{s}^{-1}\right)$. The SWNT density and length were affected by several synthesis parameters including the concentration of catalyst solution and growth temperature. The oxide layer on the Si substrate was found to promote SWNT growth. SWNTs longer than $2.5 \mathrm{~cm}$ were consistently obtained over a wide synthesisparameter window. The initial heating rate and gas flow rate had only minor effects on the SWNT length and density. The sample shown in Fig. 1 has a low nanotube density, allowing an individual SWNT to be traced by SEM imaging for length measurement.

Figure $2 \mathrm{a}$ is an atomic force microscopy (AFM) image of a 5- $\mu \mathrm{m}$ segment of the 4-cm long SWNT. To measure its diameter accurately, a height profile scan was performed across it and is shown in Fig. $2 \mathrm{~b}$. The diameter of the SWNT is equal to the height of the peak in Fig. $2 \mathrm{~b}$ and is measured as $1.4 \mathrm{~nm}$.

Raman spectra and images of SWNTs were obtained with a Kaiser Optical confocal imaging Raman microscope. The sample excitation was performed using $6 \mathrm{~mW}$ of $514.5 \mathrm{~nm}$ light with a $1 \mu \mathrm{m}$ spot size. Spacing between individual nanotubes was sufficient to allow imaging and tracking of individual nanotubes over millimetre length scales. From a sampling of 20 different nanotubes, we observed the G-peak splitting profiles and radial breathing mode (RBM) frequencies that are consistent with those of SWNTs. Both metallic and semiconducting types are present. Frequencies $(\omega)$ observed in the RBM region ranged from $110 \mathrm{~cm}^{-1}$ to $190 \mathrm{~cm}^{-1}$, corresponding to nanotube diameters $(d)$ in the range of $2.25 \mathrm{~nm}$ to $1.31 \mathrm{~nm}$ (using the expression $\omega_{\mathrm{RBM}}=248 \mathrm{~cm}^{-1} \mathrm{~nm} / d$, suitable for CVD grown nanotubes on $\mathrm{Si}$ substrates) ${ }^{6}$.

These SWNTs also displayed either very weak or no intensity in the defect mode commonly observed near $1,350 \mathrm{~cm}^{-1}$, indicating that they have relatively high structural quality. The unique Raman spectral signature obtained for each SWNT allows tracking of a single a
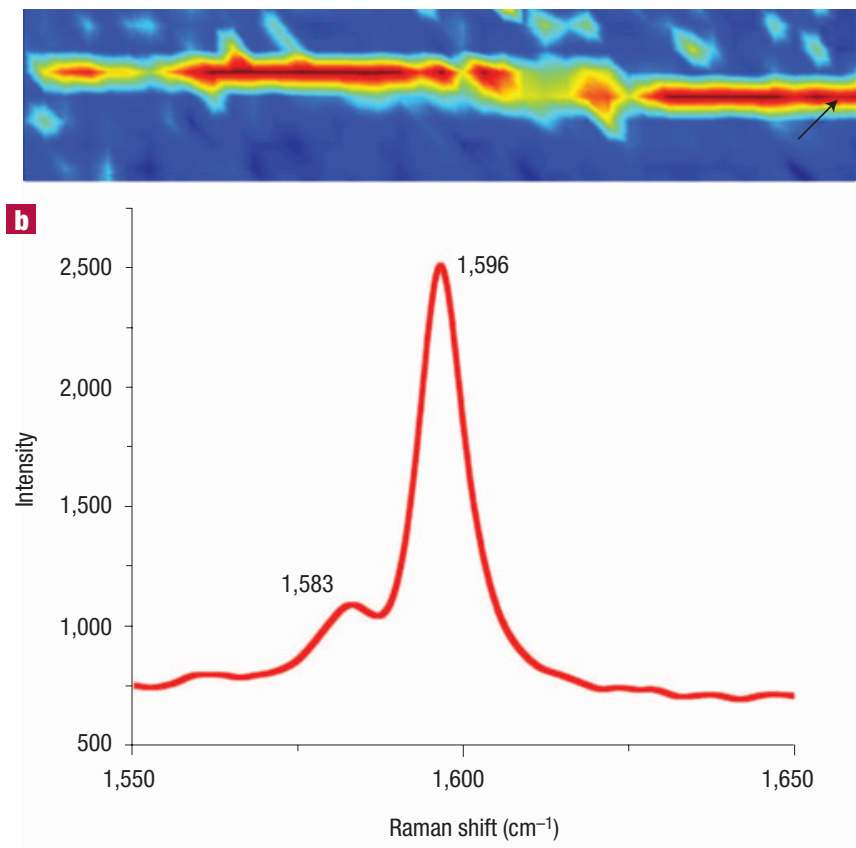

Figure 3 The Raman image and spectrum of a long SWNT.a, The Raman image is from a $20 \mu \mathrm{m}$ segment. Image intensity is obtained from integration over the G-peak region from 1,550 to $1,650 \mathrm{~cm}^{-1}$ at each sample point. b. The G-peak Lorentzian line shape and $13 \mathrm{~cm}^{-1}$ peak separation indicates this SWNT as a $1.9( \pm 0.2) \mathrm{nm}$ diameter semiconducting nanotube. This G-peak spectrum was taken from the location on the SWNT marked by a black arrow in $\mathbf{a}$.

nanotube over large length scales. We tracked a long SWNT spectrally for $2 \mathrm{~mm}$, with its typical image and G-peak spectrum shown in Fig. 3. Each point in the image (Fig. 3a) represents a complete Raman spectrum and permits evaluation of the stability of the nanotube structure over its sampled length. The spectrum shown in Fig. $3 \mathrm{~b}$ is preserved over the full $2 \mathrm{~mm}$ probed for this SWNT, with minor $\left(<2 \mathrm{~cm}^{-1}\right)$ shifts in frequency, which indicates that the diameter change, if it exists, is less than $1.5 \%$ over the probed $2 \mathrm{~mm}$ length.

Thelong SWNTs appear to grow by a tip-growth mechanism, that is, the catalytic Fe particles moved with the growing SWNT tips. A basegrowth with a stationary catalyst particle would require the whole SWNT to overcome the van der Waals forces with the substrate and slide on the substrate, which is clearly impossible ${ }^{7}$. Only the tip-growth mechanism could result in an ultralong SWNT lying on a substrate. Some SWNTs were found terminated on the substrate surface away from the edges, with ending segments usually several hundred micrometres long. They are observed to be usually wavy and tangled with themselves, as shown in Fig. 4, even though their other segments are straight. In contrast, the 4-cm long SWNT is relatively straight along most of its length (see the Supplementary Information). Therefore, it appears that once an SWNT becomes wavy, its growth soon stops. Note that the wavy growth was not caused by a local disturbance of the flowing gas, because straight SWNTs also grew near wavy SWNTs (see Fig. 4).

It appears that the wavy SWNT growth and the eventual growth termination were caused by the 'skidding' of the catalyst particle on the Si substrate surface. A mechanism has been proposed for long SWNT growth on a substrate ${ }^{8}$, assuming that the catalyst particle floats above the substrate and that segments of a long SWNT lie on the substrate 


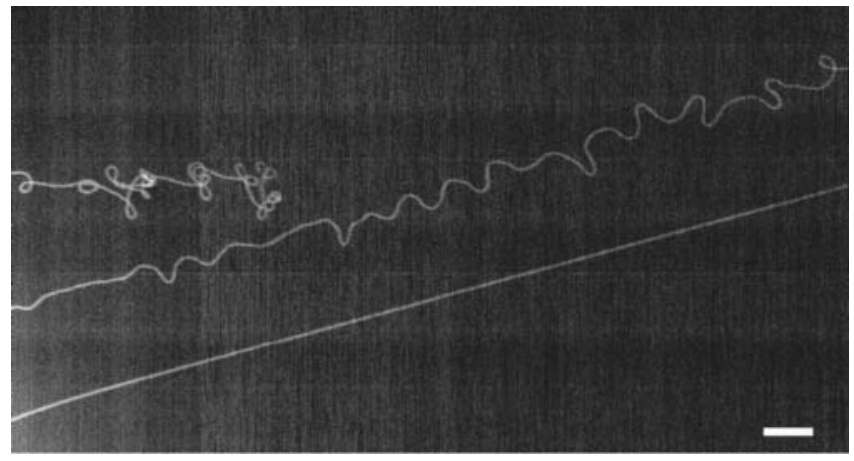

Figure 4 Morphology of SWNTs that ended on the Si Substrate surface away from edges. The SWNT on the top is wavy and tangled before it ends on the substrate. The SWNT in the middle is starting to become wavy and will terminate on the substrate after growing several hundred micrometres longer. The SWNT at the bottom is straight and continues to grow to longer length. The scale bar is $10 \mu \mathrm{m}$.

after their formation due to the van der Waals forces. We believe that the same mechanism also governs the growth of ultralong SWNTs in this study. However, due to the low flow rate of the carrier gas $\left(\mathrm{Ar}+6 \% \mathrm{H}_{2}\right)$ in this experiment, the catalyst particle was expected to float just slightly above the Si substrate. When a catalyst particle came into contact with the substrate surface due to flow perturbation or for other reasons, it could skid on the substrate surface, during which it would change its composition, morphology, and size due to interactions with the substrate and/or with other impurities accumulated on the substrate. At the synthesis temperature of $900{ }^{\circ} \mathrm{C}$, the iron catalyst particle can pick up a significant amount of the $\mathrm{Si}(\sim 10$ at.\%) without changing crystal structure according to the equilibrium phase diagram ${ }^{9}$. The nanosized Fe particle is expected to have even higher Si solubility ${ }^{10}$. When the frictional force increases to where the catalyst particle cannot move fast enough to accommodate the SWNT growth along the gas flow direction, the SWNT may grow backwards to form the wavy SWNT (see Fig. 4). As shown, the waviness becomes worse with the SWNT growth along the gas flow direction due to increasing frictional forces as the catalyst particle gradually changes its characteristics during its skidding across the substrate. After skidding for more than $100 \mu \mathrm{m}$, the catalyst particle finally became inactive and the SWNT growth stopped. The termination mechanism discussed above is different from those of multiwall nanotubes with much larger catalyst particles ${ }^{11,12}$.

Raman imaging of SWNTs that terminated before the substrate edge also provides some insight into the termination mechanism. Shown in Fig. $5 \mathrm{a}$ is a Raman image of the tip of a prematurely terminated SWNT. This is a different long SWNT from those shown in Figs 1, 3 and 4 . The tip image is much larger than the $1 \mu \mathrm{m}$ spatial resolution of our instrument and displays a very high intensity. Additionally, as seen in Fig. 5b, multiple radial breathing modes (at 110, 130,145 and $171 \mathrm{~cm}^{-1}$ ) are now present, compared with the single mode at $171 \mathrm{~cm}^{-1}$ observed over most of the length of this particular SWNT. These observations are consistent with the nucleation of dense nanotubes on the catalyst tip. This is further evidence to support our earlier assertion that growth termination results from a change in catalyst particle characteristics that could inhibit further growth of the single ultralong SWNT.

The parameters that could be optimized for growing longer SWNTs include the flow rate and composition of the carrier gas, the growth temperature, and the initial concentration of the catalyst solution. The fact that all long SWNTs observed in this study fall in a diameter range of $1.31 \mathrm{~nm}$ to $2.25 \mathrm{~nm}$ suggests that the catalyst particle size played
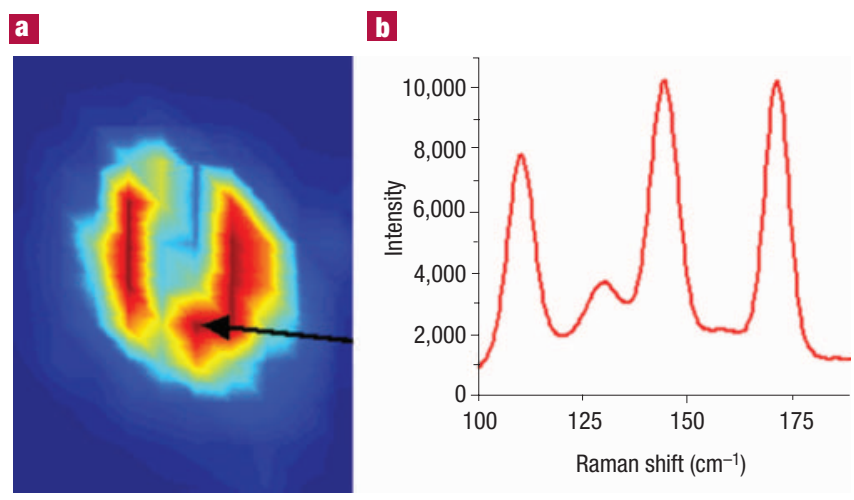

Figure 5 Raman image and spectrum of the growth termination region of a long SWNT indicates nucleation of dense nanotubes. a, Image of nanotube tip region. The image width is $5 \mu \mathrm{m}$. Image intensity is from integration over the G-peak spectral region. $\mathbf{b}$, Radial breathing mode region of spectrum obtained at the image point shown by the arrow.

an important role in the growth of ultralong SWNTs. The particle size is affected by the initial concentration of catalyst solution. Both the particle size and the carrier-gas flow rate will affect the floating of the catalyst particle, and hence the SWNT growth. Ethanol seems critical for the growth of ultralong SWNTs, probably because it does not tend to form amorphous carbon on dissociation ${ }^{13}$.

Our synthesis of a 4 -cm-long individual SWNTs on a Si substrate indicates the possibility of synthesizing continuous SWNTs without any length limitation. The extremely high growth rate of $11 \mu \mathrm{m} \mathrm{s}^{-1}$ make them very attractive for commercial applications. Previous reports of long nanotube structures have been limited to yarns ${ }^{3}$, strands $s^{4,14}$ and fibres ${ }^{15}$ formed from much shorter individual nanotubes or nanotube-polymer matrix composite fibres ${ }^{16-19}$. The 4 -cm-long individual SWNTs reported here will significantly impact a wide spectrum of applications including electronic devices, microelectromechanical systems, bio-sensors, scaffolding for neuronal growth $^{20}$, robotics, space exploration, personal armour, and sporting goods.

Received 7 May 2004; accepted 30 July 2004; published 12 September 2004.

References

1. Iijima, S. Helical microtubules of graphitic carbon. Nature 354, 56-58 (1991).

2. Baughman, R. H., Zakhidov, A. A. \& de Heer, W. A. Carbon nanotubes-the route toward applications. Science 297, 787-792 (2002).

3. Jiang, K. L., Li, Q. Q. \& Fan, S. S. Spinning continuous carbon nanotube yarns. Nature 419, 801 (2002).

4. Zhu, H. W. et al. Direct synthesis of long single-walled carbon nanotube strands. Science 296, 884-886 (2002).

5. Huang, S., Maynor, B., Cai, X. \& Liu J. Ultralong long, well-aligned single-walled carbon nanotube architectures on surfaces. Adv. Mater. 15, 1651-1655 (2003).

6. Dresselhaus, M. S., Dresselhaus, G., Jorio, A., Souza Filho, A. G. \& Saito, R. Raman spectroscopy on isolated single wall carbon nanotubes. Carbon 40, 2043-2061 (2002).

7. Huang, S., Cai, X. \& Liu J. Growth of millimeter-long and horizontally aligned single-walled carbon nanotubes on flat substrates. J. Am. Chem. Soc. 125, 5636-5637 (2003).

8. Huang, S., Woodson, M., Smalley, R. E. \& Liu, J. Growth mechanism of oriented long single walled carbon Nanotubes using "fast-heating" chemical vapor deposition process. Nano Lett. 4, 1025-1028 (2004).

9. Brandes, E. A. \& Brook, G. B. (eds.) Smithell's Handbook 7th edn, Ch. 11, 271 (ButterworthHeinemann, Oxford, UK, 1992).

10. Suryanarayyana C. (ed.) Non-equilibrium Processing of Materials 59 (Pergamon, Amsterdam, 1999).

11. Liao, X. Z. et al. Effect of catalyst composition on carbon nanotubes growth. Appl. Phys. Lett. 82, 2694-2696 (2003).

12. Dai, H. et al. Single-wall nanotubes produced by metal-catalyzed disproportionation of carbon monoxide. Chem. Phys. Lett. 260, 471-475 (1996).

13. Maruyama, S., Kojima, R., Miyauchi, Y., Chiashi, S. \& Kohno, M. Low-temperature synthesis of high 
purity single-walled carbon nanotubes from alcohol. Chem. Phys. Lett. 360, 229-234 (2002).

14. Wei, J., Jiang, B., Wu, D. \& Wei, B. Large-scale synthesis of long double-walled carbon nanotubes. J. Phys. Chem. B 108, 8844-8847 (2004).

15. Li, Y. L., Kinloch, I. A. \& Windle, A. H. Direct spinning of carbon nanotube fibers from chemical vapor deposition synthesis. Science 304, 276-278 (2004).

16. Andrews, R. et al. Nanotube composite carbon fiber. Appl. Phys. Lett. 75, 1329-1331 (1991).

17. Vigolo, B. et al. Macroscopic fibers and ribbons of oriented carbon nanotubes. Science 290, 1331-1334 (2000).

18. Kumar, S. et al. Synthesis, structure, and properties of PBO/SWNT composites. Macromolecules 35, 9039-9043 (2002)

19. Dalton, A. B. et al. Super-tough carbon-nanotube fibres. Nature 423, 703 (2003).
20. Hu, H., Ni., Y., Montana, V., Haddon, R. C. \& Parpura, V. Chemically functionalized carbon nanotubes as substrates for neuronal growth. Nano Lett. 4, 506-511 (2004).

Acknowledgements

This work was supported by the Laboratory Directed Research and Development program office of the Los Alamos National Laboratory.

Correspondence and requests for materials should be addressed to Y.T.Z.

Supplementary Information accompanies the paper on http://www.nature.com/naturematerials

Competing financial interests

The authors declare that they have no competing financial interests. 